\title{
nan0sphere: Location-Driven Fiction for Groups of Users
}

\author{
Kevin Eustice, V. Ramakrishna, Alison Walker, \\ Matthew Schnaider, Nam Nguyen, Peter Reiher \\ University of California, Los Angeles \\ kfe@cs.ucla.edu
}

\begin{abstract}
We developed a locative fiction application called nanOsphere and deployed it on the UCLA campus. This application presents an interactive narrative to users working in a group as they move around the campus. Based on each user's current location, previously visited locations, actions taken, and on the similar attributes of other users in the same group, the story will develop in different ways. Group members are encouraged by the story to move independently, with their individual actions and progress affecting the narrative and the overall group experience. Eight different locations on campus are involved in this story. Groups consist of four participants, and the complete story unfolds through the actions of all four group members. The supporting system could be used to create other similar types of locative literature, possibly augmented with multimedia, for other purposes and in other locations. We will discuss benefits and challenges of group interactions in locative fiction, infrastructure required to support such applications, issues of determining user locations, and our experiences using the application.
\end{abstract}

\section{Introduction}

Our work on ubiquitous computing has focused on group interactions among users and devices. One important element of this work has been to deliver useful and interesting content to users based on their physical locations, often modulated by the social groups to which they belong. We built a sample application based on applying these ideas to stories that are presented to users based on their location. This paper discusses the design of our system, important issues we addressed in building the application, and experiences in its use in a real environment, the UCLA campus.

We built a story called nanOsphere that would play out across several locations on the UCLA campus. Because of our interest in group interactions, we designed it as a story told to a small group of users.

Members of this group take on different story roles and move from place to place, either individually or together. The story unfolds as they visit different locations, and depending on the participation of other group members, the plot may vary. It can only reach its conclusion if all members participate and all locations are visited.

Though the text of the story itself is particular to a single narrative, the software infrastructure used to present it to the groups of users is general and would support many other pieces of literature or other forms of content with similar characteristics. Common infrastructural elements include methods of determining location, a parser to convert raw text and interaction logic into material to present to the readers, primitives to handle group formation and interactions, and networking and event 
communications support. We also addressed how caching and cooperation can be used to handle problems arising from poor or intermittent network connectivity.

We discuss not only the issues of the story we actually wrote and tested, but also the generality of the system and its usability for a wider variety of locative media. The experiences we present include discussions both of the difficulties in writing this kind of story and in debugging and experiencing it.

\section{System Support for Location-Based Interactive Narratives}

Our narrative framework allows authors to deploy content and dynamically provides services to individual mobile users and groups of users. Our Panoply middleware supports the creation and management of decentralized groups of computing entities called spheres of influence, which serve as an organizing principle for applications. In our target environment, story participants carry mobile devices that represent them in cyberspace. Panoply enables the formation of spheres and provides tools for application design and content authoring. These tools were designed to be useful for a wide variety of interactive experiences involving groups and locations.

\subsection{Story-Telling Application}

nan0sphere, was designed to be an interactive, location-and character-driven work of speculative fiction that involved a wide variety of different physical locations on our campus. nanOsphere is a non-linear, team-based narrative in which each member assumes the role of a story character. They each receive a customized experience, which includes pieces of the story and clues, based on their location, the location of other team members, and the overall progress of the narrative. Each user has the opportunity to manipulate virtual objects and perform virtual actions that change the state at the present location. No individual player is exposed to the whole narrative; only by combining the team's individual experiences does the entire narrative emerge. nanOsphere requires the creation and management of groups of entities. These could be location-based groups, such as all the characters who happen to be in the library at a given time. Social relationships and shared tasks or experiences could also define a group. For our application, we created a virtual social group that maintained story state and was responsible for disseminating content. This social group is represented by a network server; mechanisms for on-demand communication of story events and delivery of locative media content are provided as core features. Discovery of peers and mediation of interactions between two participants, or a participant and a virtual location, were supported. These features are common to many applications, independent of the content or the nature of the participants.

Media Language and Parser: For the creation and interpretation of locative media content, we provided a media description language based on XML that consists of basic media elements such as location descriptions, conditional scenarios, and actions that users may take. Additionally, we provided social conditionals that let the media engine test various constraints such as the presence of another team member or the presence of someone exploring a different media piece. Figure 1 shows an example of 
a location description. This example shows a simple location and also illustrates a triggerable scenario that is activated when the participant is playing the "Rowan" character, and the "Renata" character is also present in the vicinity.

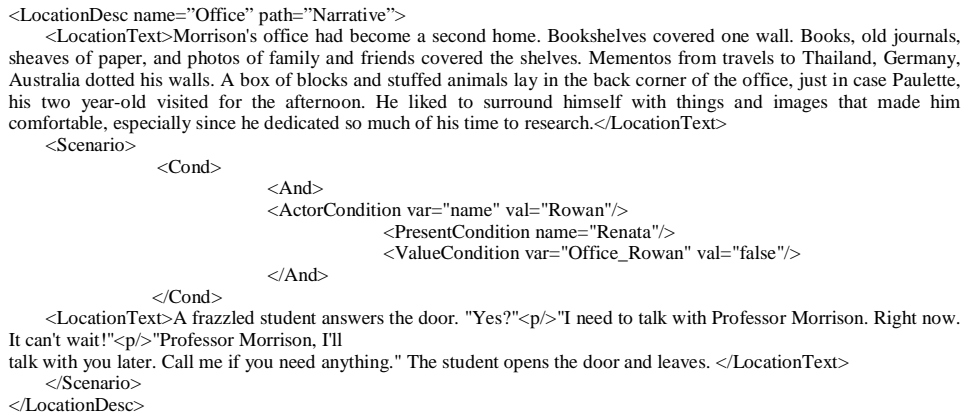

Figure 1: Example Locative Media Description

User Interface: nanOsphere presents users with a Java-based interface (Figure 2). The interface presents a portion of the story to the participant on his mobile device; this content is relevant to his current position, and reflects the progress of the story based on actions performed by all the characters. In the figure, the user has assumed the role of William, one of the story characters, who has just arrived at the Inverted Fountain. The main panel, marked "Location Description," displays relevant story text, laying out the scene as it would appear at the virtual Inverted Fountain. The interface also indicates important events that provide the user with more information and also supplies contextual hints or prompts in an "Event Log" panel. The user can alsc influence the course of the story through actions; choices for these are provided in an "Available Actions" panel. As the user moves around campus and enters other locations, the interface automatically updates the scene description and the available options.

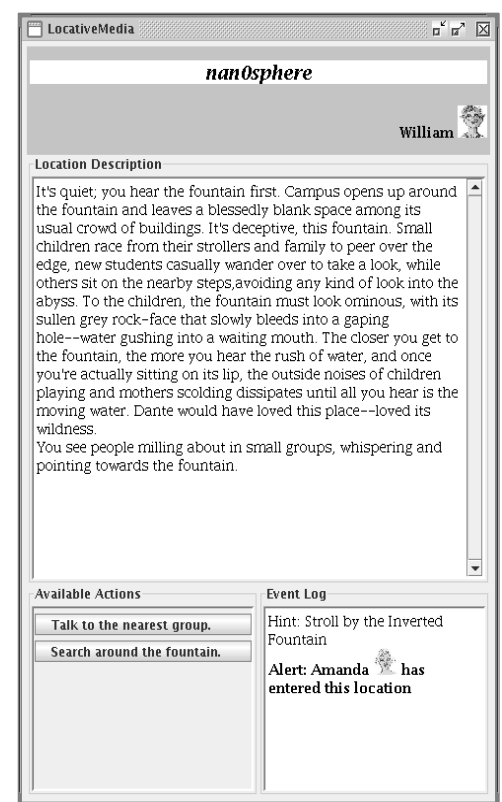

Figure 2: nanOsphere's User Interface 


\subsection{The Panoply Middleware}

Applications such as nan0sphere run on top of Panoply, our ubiquitous computing middleware, which handles location inference, networking and configuration, group context management, and communication with other devices and services.

Location Inference: In mobile story-telling applications, location is an important component in determining what part of the story is immediately relevant to the user. Panoply provides a localization module for sensing of semantic locations, which are regions that are meaningful to users or applications. Semantic locations are obtained by mapping low-level hardware observables to semantic identifiers. The framework is modular to allow the use of different low-level localization techniques [5,10]. Our current implementation uses a combination of 802.11 scene analysis [1] and attenuation monitoring. The semantic localization component reports when a user is inside or outside of a particular office or when a user is likely within visual proximity of a relevant campus landmark. These semantic regions can be defined over a wide range of sizes, and can be subdivided or aggregated into other related semantic zones.

Network Configuration: Devices participating in an interactive narrative application need to dynamically retrieve content and maintain relationships to peers and story groups. Administrative realities do not permit deployment of content servers at every location. As users explore the campus with their mobile device, Panoply monitors the wireless landscape to identify appropriate 802.11 networks. Based on configuration information provided by the nanOsphere application, Panoply manages network and media server connectivity. In practice, Internet connectivity is not always available. Where no connectivity is available, ad hoc 802.11 networks are used to discover and form connections to nearby peers in order to allow local coordination and interaction.

Groups-Based Infrastructure: In Panoply, groups and group connectivity are managed by Spheres of Influence [9], a device management and coordination system. These spheres are based on user and device characteristics such as social memberships, location, network presence, etc. Panoply provides low-level primitives, including group creation and discovery. Spheres maintain policy, state, memberships and relationships, provide contextual sensors, and securely mediate interactions.

A sphere represents a single device, or, recursively, a structured group of spheres. Most groups occurring in mobile story-telling applications will fit in one of the following classes: device spheres, location spheres, and social or attribute-based spheres. A device sphere represents a single mobile device. A location sphere is associated with describable physical regions, such as a room, building, or the area within range of a specific access point, and can include any device spheres currently in that space. Social spheres represent groupings of other spheres to achieve tasks or indicate common interests or goals, such as being members of a club. All spheres are maintained by one or more devices and have a network presence. An example in our application is an interactive narrative sphere, a type of social sphere. The participants' mobile devices have device spheres, and are transient and peripatetic members of the narrative sphere.

Event Communication: Panoply uses a publish-subscribe event model for communication, which is well suited to the loosely coupled mobile computing model 
we are building our applications on. Events can be used to deliver low-level context changes and notifications, as well as on-demand content. Sphere components and applications register with their local sphere for desired event types, and corresponding events are delivered to the interested component. System events include discovery, membership, location, policy, cache, heartbeat, and management events, etc. These events are generated by core Panoply components. Applications use these events to react to external changes and adapt their behavior. Application-defined events are specific to the creating application, e.g., nanOsphere uses media-update events from the media sphere to the mobile devices, and action events from mobile devices to the media sphere.

Media Caching: Various locations that are critical to developing the story may have poor or no connectivity, yet provide sufficient information to allow a mobile device to determine the identity of that location. Therefore, we enable predictive delivery of content from the social media sphere to individuals' devices during periods of connectivity. This content is then stored in a sphere cache. Then, if the media sphere is disconnected and our location subsystem indicates we have entered a new location, we can check to see if the cache contains appropriate media. If it does, the local infrastructure reveals the content to the application. Additionally, changes to virtual story state made by the local device are cached until connectivity is restored, or may be shared with locally discovered team members if any exist.

\section{The nan0sphere Locative Media Experience}

nan0sphere is an interactive and location-aware narrative, written by a UCLA graduate student in the English department and two undergraduates (one in English and one in Computer Science). The goal of this project was to showcase group interactivity and location-aware media, and at the same time, tell a story.

The story is a speculative, fictional narrative about nanotechnology on the UCLA campus. Four users each play a different character (a security guard, a graduate student, a campus information technology specialist, and a professor of sociology) and interact with the campus from that person's perspective. The narrative is goaldriven, and uses this concept as the impetus for each character to move from location to location.

The authors used the construction of a new biotechnology building on UCLA's campus as the main plot-device for the narrative. The story begins with the theft of an extremely dangerous prototype technology from a campus nanotechnology lab. Specifically, the story takes the four users through eight specific points on the campus. They are able to read descriptions of the surroundings as they stand in a location. As the character visits more locations, the true story behind the theft is revealed. Each character has a different reason for being involved in the story: for example, the graduate student might lose her funding, while the sociology professor is best friends with the head of the lab that was broken into.

The story has a definite plot arc, with each player entering at the "beginning" of his or her involvement in the story. Players gather clues at each location and can engage in virtual conversations with other characters. Multiple players in a single location or two players crossing paths can lead to more of the story being revealed or to the clues 
changing. Players are also encouraged to engage in actual conversation and discuss the narrative if they happen to cross paths.

In addition to the exploration of the central storyline of nanOsphere, the authors wanted to create more conceptual media experiences for users using the same story. Based on the same locations, the authors created three other alternative paths that users could take, allowing them to experience the same story from various, and often unexpected, points of view. The paths use different forms of narrative, ranging from poetry and song to drama and prose, freely quoting other authors in order to form complex layers of experience. The nanite path follows the stolen swarm of nano-scale robots as they gain sentience and awareness; the future path considers the UCLA campus in a post-nanite world; the Wesley path follows the thief who originally stole the technology, and his descent into madness. It is possible to switch back and forth between these three paths. The authors also wanted to create an "infectious" paradigm within the story. When any of the four players come close to being infected with the stolen nanites, they can "jump" to the nanite path for a moment as a way to suggest infection; the same would occur if any of the players accidentally came too close to Wesley: they can choose to enter his path and explore his mind.

\section{Lessons from nan0sphere}

Our experience with the deployment has yielded interesting insights into design issues for locative media and locative media infrastructure, as well as issues and questions for authors developing location-aware media. The relationship between software author and storyteller is significantly blurred as infrastructural limitations feed into the narrative, and narratives approach the level of software in their complexity.

Social Issues in nan0sphere:, It became clear that the storyline's dependencies on coordinated actions taken by multiple characters could be problematic. The story could only progress when different characters took specific actions, pushing forward the story's progress. At a given moment, any given character might find that they had no options in any story location as the story was blocked, waiting for some other character to make progress. If a participant took a break from the story, he could effectively prevent other characters from accessing new content and completing the narrative. Depending on author intent, this might be not acceptable. One possible solution would be to implement narrative event timers that ensure the narrative advances at a reasonable rate by triggering unresolved game events necessary for active characters to progress. From a larger perspective, the infrastructure should not always be forcing narrative progress. A content author might in fact want to require one character to wait upon another's actions without any other narrative recourse.

Debugging Interactive Narratives: While refining our framework, we built a number of debugging tools. We found that it was desirable to exercise the application without actually moving about the campus, and thus we created a clickable map of the campus to simulate location transitions. Additionally, we built a version of our media interpreter that displayed and logged the conditional decisions affecting the current story. In our experience, it would be useful to have a comprehensive debugging 
framework so that developers and authors could easily isolate narrative components and test them under both real and simulated conditions.

For example, in the narrative description language, the authors were able to specify what text they wanted to associate with various locations. They were also able to specify various conditions that controlled when certain portions of the text were made available for display. During testing, it became evident that the authors did not, and with available tools could not, completely anticipate all possible paths that individual characters could take. On occasion an individual user's experience might include character introduction or plot development that seems to be "out of order," at least to the extent that text in interactive media can be so. Clearly, we need better support for authors to express high-level flow constraints on their stories, akin to software invariants. One role of a debugging framework could be constraint verification on the narrative content, to point out possible narrative flow problems to the author.

Localization Issues: The nan0sphere authors selected eight locations on campus to be semantic regions that play a part in the narrative, prior to the implementation of our localization code. Some of the referenced locations are highly specific, intending that the user be in one small area, such as an individual room, or a particular bench in a garden. Others are intended to be more broadly defined and aim to have the user in the general proximity of a landmark. In both cases, accurate localization is important. In the former case, we wanted to be somewhat forgiving how about how precisely a user had to be in a particular location. Users might be discouraged if they go to an area specified in the story, but are unable to situate themselves in the exact position the authors envisioned. By defining a slightly larger zone, users need only approach the general area to know that they are on the right track. Three of the locations chosen are particularly close to one another. Two of these are outdoor locations and one is indoor. Although these regions do not overlap, they are close enough that it can be difficult to absolutely differentiate one from another, clearly presenting difficulties for participants. This is a limitation of our localization technique; however, in general, some limitations will exist in many localization schemes. Content authors need to be aware of the limitations of the localization support and design accordingly.

When the device determines that it has moved to a new location, our prototype gives both auditory and visual cues. The auditory cue was added during debugging, and though it is configurable, we have typically left it enabled. We discovered that users tend to focus their attention on their devices when they changed location, possibly as a result of the tone. The change in location results in a corresponding change of text displayed to the user. Users tended to immediately read the new text and proceed with the story directly from that location. Thus, in the cases where the user was supposed to reach a specific point, they sometimes did not get to the authors' exact intended location before progressing with the story. It may be possible to modify the interface to inform users when they are getting "warmer" so as to lead them all the way to the intended location before allowing the story to progress.

Authorial Issues: From the authors' perspective, nanOsphere was difficult to write for two reasons: First, it is always hard for three individuals with different levels of expertise in creative writing, and especially creative writing within a new media framework, to come together with cohesive ideas and execute them in a manner that is fair to all involved. Second, two of the writers did not have much expertise in computer science, which made it hard to understand how to use and showcase the 
features of Panoply. An important and difficult question for future collaborations between technologists and artists is which should come first, the making of the software-in itself an artistic process-or the creative components of locative storytelling?

This question is not easily answered, other than to leave locative narratives to those who are adept at both technological and artistic pursuits. This answer is unsatisfactory to most people pursuing media projects, and overlooks the rich tradition of collaboration within new media and electronic literature. Electronic literature that uses innovative interfaces and novel means of communication is often a collaboration between artists and programmers. As Strickland and Lawson, the creators of Vniverse [17] suggest, their project "could not have existed as an individual project, and we find that we most enjoy performing it in collaboration as well." We agree with Lawson and Strickland: true collaboration between artists and technologists occurs when the project is conceived by both parties.

Another challenge was how to engage the reader to want to walk around campus. It is easy to keep the users' attentions when locative media is performed in a small space; how does an author capture the users' interest enough for them to trek through a mile-long campus? nanOsphere's narrative "bounced" between physically distant locations on the UCLA campus. To fully explore the story, participants traveled back and forth between different story locations. Sometimes a character would arrive at a new location, only to be told to go back to the last location she visited. Unless the focus of a narrative is to encourage exercise, forcing user mobility can be tedious. If a narrative is to effectively influence a participant to change locations, the narrative must offer sufficient allure to overcome human inertia. A very compelling narrative, or some form of competition and reward may be sufficient.

The decision was made early to make nan0sphere a plot-driven mystery and use clues and cliffhangers that propel the narrative forward and encourage people to walk around the campus to try and find more clues. Experience with running users through the story suggested that this approach was not sufficient for the amount of user movement the story demanded. Perhaps a narrative designed to serve as a tour of an interesting area could solve this problem. One promising possibility to avoid too much user movement is to restructure the notion of locations in the locative media. Events in locative media may not be tied to a specific location, e.g. "Café Roma," but rather to a type of location, e.g. a café or restaurant, etc., or location template. Using this technique, a locative narrative might progress as the participant goes about their daily routine, only forcing particular movements for major story events.

The project's conceptual layers (the different "paths" one can take to reveal more of the story) were a response to the artistic constraints that such a plot-driven story implied. They enabled the authors to experiment with prose styles and use the software in novel ways with regards to location and user interaction. The authors needed to agree on what kind of story they should construct and who their intended audience was. The group vacillated between wanting to present their audience with a very abstract media experience that worked with the same concepts (nanotechnology, the body, the relationship between scholar and subject), but relied on users to draw their own connections as to how they would navigate through the project, and a straightforward narrative that presented a "real" story, one with reasons behind every action. Here is the fundamental divide that was encountered when creating nan0sphere: what constitutes a real and maybe more importantly enjoyable story? It 
proved difficult to create a narrative that was exciting conceptually, yet concrete enough so that users would feel they were really getting somewhere.

\section{Related Work}

Mobile Bristol [12] and InStory [2] take a toolkit-based approach towards supporting the authoring of locative media, similar to Panoply. Mobile Bristol focuses on enabling rapid authoring of locative media contents, or mediascapes, on Windowsbased PCs and palmtops. InStory provides an authoring environment that supports mobile storytelling, gaming activities and access to relevant geo-referenced information through mobile devices such as PDAs and mobile phones. The infrastructure provides localization services, as well as relevant media encoded in $\mathrm{XML}$, as does Panoply. InStory also enables explicit interactions among users through GUIs. Mobile Bristol and InStory primarily focus on enabling easy content development by authors who have limited programming skills, Similarly, the iCAP [7] toolkit allows users more control over how their designed applications behave without having to write code, though it provides no infrastructural features like localization. We add to the richness of the experiences that can be created by such toolkits by treating groups as first-class primitives in the Panoply infrastructure, and make group interactions implicit. Panoply also manages dynamic network selection and configuration, a hard problem that is crucial to the success of mobile applications.

The fields of social entertainment (Ghost Ship [11], Pirates! [3], SeamfulGame [4], CitiTag [15]) and museum tours [6][8][14][16] have done much to enhance user experience through locative media. Users can play games or gain knowledge about the objects in their immediate environment through interfaces on their mobile devices. But many of these systems do not provide the level of interactivity and freedom of movement that Panoply-based applications do. Even those applications that are more interactive than the others [6] are not cannot be generalized beyond their immediate application, do not support user-specific customization, and are not group-aware.

\section{Conclusions}

The success of nanOsphere is mixed. We learned much about the realities of building this kind of locative media application, and it helped improve the Panoply infrastructure. Some of tools built in conjunction with nanOsphere may be helpful in building other such applications, and the lessons that we learned will benefit other groups. On the other hand, nan0sphere did not become popular. Even members of our own group found working through the entire story somewhat tedious, and there was no enthusiasm for running through multiple story outcomes or exercising optional features, in large part because of the amount of physical movement required. Perhaps the single greatest lesson that this application offers is that peripatetic stories require strong motivations for the movements they require. A story must be extremely compelling to get its readers to walk up and down hills, go into and out of several buildings, and figure out exactly which locations need to be visited next. 
An important lesson in regards to the group aspects of nanOsphere is that the group experience must be designed to involve the group, yet not require too stringently that all group members participate at once or experience the story at the same speed. This offers extra challenges in designing such stories.

From a technical point of view, fixing an exact location is often difficult. While technologies like GPS would handle some of our difficult situations well, those technologies have their own weaknesses and challenges. Storytellers using these technologies must keep these limitations and inaccuracies in mind, both when choosing locations and determining how to ensure that their stories make progress.

Designing and supporting a good peripatetic story is not easy. There are major challenges in conceiving the story, in providing technology that supports its needs, and with ensuring that the experience meets the desires of one's audience. Much work will be required to make this form of storytelling easy to create (or, at least, as easy as writing any good story can be) and enticing to its audience.

\section{References}

[1] Bahl, P., and Padmanabhan, V. N.: "Radar: An In-Building User Location and Tracking System," Proceedings of IEEE Conference on Computer Communication, Vol. 2.

[2] Barrenho, F., Romao, T., Martins, T, and Correia, N.: "InAuthoring environment: interfaces for creating spatial stories and gaming activities," In Proceedings of the 2006 ACM SIGCHI Intl. conference on Advances in Computer Entertainment Technology, Hollywood, CA.

[3] Bjork, S., Falk, J., Hansson, R., and Ljungstrand, P.: "Pirates! - Using the Physical World as a Game Board," In Proceedings of Interact 2001. Tokyo, JAPAN. (July 2001)

[4] Borriello G., Chalmers M., LaMarca A., and Nixon, P.: "Delivering Real-World Ubiquitous Location Systems," Communications of ACM, Vol. 48, No. 3. (March 2005) 36-41

[5] Capkun S., Hamdi M., and Hubaux J., "GPS-Free Positioning in Mobile Ad Hoc Networks," Proceedings of Hawaii Int. Conference on System Science, January 2001.

[6] Chou, S.-C., Hsieh, W.-T., Gandon, F., and Sadeh, N.: "Semantic Web Technologies for Context-Aware Museum Tour Guide Applications," Proc. WAMIS 2005. (March 2005)

[7] Dey, A. K., Sohn T., Streng S., and Kodama J., "iCAP: Interactive Prototyping of ContextAware Applications," Proc. Fourth Intl. Conference on Pervasive Computing, 2006

[8] eDocent Website http://www.ammi.org/site/extrapages/edoctext.html

[9] Eustice K., Kleinrock L., Markstrum S., Popek G., Ramakrishna V., and Reiher P., "Enabling Secure Ubiquitous Interactions," Proceedings of 1st International Workshop on Middleware for Pervasive and Ad Hoc Computing (co-located with Middleware 2003)

[10] Kaplan, E., Understanding GPS, Artech House, 1996.

[11] Hindmarsh, J., Heath, C., vom Lehn, D., Cleverly, J.: "Creating Assemblies: Aboard the Ghost Ship," Proc. 2002 ACM Conference on Computer Supported Cooperative Work.

[12] Hull, R., Clayton, B., Melamed, T.: "Rapid Authoring of Mediascapes," In Proceedings of Ubicomp 2004.

[13] Kindberg, T., Barton, J., Morgan, J., Becker, G., Caswell, D., Debaty, P., Gopal, G., Frid, M., Krishnan, V., Morris, H., Schettino, J., Serra, B., Spasojevic, M.: "People, Places, Things: Web Presence for the Real World," Mobile Networks and Applications, V.7, issue 5.

[14] Kwak, S.Y.: "Designing a Handheld Interactive Scavenger Hunt Game to Enhance Museum Experience," MA Thesis. Michigan State University. (2004)

[15] Quick, K., Vogiazou, Y.: CitiTag Multiplayer Infrastructure, TR: KMI-04-7 (March 2004)

[16] Schmalstieg, D. and Wagner, D.: "A Handheld Augmented Reality Museum Guide," In Proc. IADIS Intl Conf. on Mobile Learning (ML2005). Qawra, Malta. (June 2005)

[17] Strickland, C. and Lawson, C.: "Making the Vniverse," http://www.cynthialawson.com/vniverse/essay/index.html. 